\title{
EXTRACTION OF RIVERS AND LAKES ON TIBETAN PLATEAU BASED ON GOOGLE EARTH ENGINE
}

\author{
R.G. Xu, G. Qiao*, Y.J. Wu, Y.J. Cao
}

\begin{abstract}
College of Surveying and Geo-Informatics, Tongji University, Siping Road 1239, Shanghai 200092, China - xrg@tongji.edu.cn; qiaogang@tongji.edu.cn; 1633318@tongji.edu.cn; yogacao@tongji.edu.cn.
\end{abstract}

Commission III, WG III/9

KEY WORDS: Landsat Images, Tibetan Plateau (TP), Water Extraction, Time Series Analysis, Google Earth Engine(GEE)

\begin{abstract}
:
Tibetan Plateau (TP) is the most abundant area of water resources and water energy resources in China. It is also the birthplace of the main rivers in Southeast Asia and plays an important strategic role. However, due to its remote location and complex topography, the observation of surface hydrometeorological elements is extremely scarce, which seriously restricts the understanding of the water cycle in this area. Using remote sensing images to extract rivers and lakes on TP can obtain a lot of valuable water resources information. However, the downloading and processing of remote sensing images is very time-consuming, especially the processing of remote sensing images with large-scale and long time series often involves hundreds of gigabytes of data, which requires a high level of personal computers and is inefficient. As a cloud platform dedicated to data processing and analysis of geoscience, Google Earth Engine(GEE) integrates many excellent remote sensing image processing algorithms. It does not need to download images and supports online remote sensing image processing, which greatly improves the output efficiency. Based on GEE, the monthly data of Yarlung Zangbo River at Nuxia Hydrological Station and the annual data of typical lakes were extracted and vectorized from the pre-processed Landsat series images. It was found that the area of Yarlung Zangbo River at Nuxia Hydrological Station varies periodically. The changing trend of typical lakes is also revealed.
\end{abstract}

\section{INTRODUCTION}

As the "Asian Water Tower", the TP is the birthplace of many famous rivers in Asia, which has a close impact on water supply, irrigation and flood forecasting in surrounding countries(Huang et al., 2018a; Huang et al., 2018b). And there are many lakes on TP, in a total of more than 1500 . Most of them are salty lakes formed by the convergence of inland rivers into basins. The unique geographical location and abundant water resources of TP make it highly sensitive and significant to the climate change in the surrounding areas. The change of surface water resources is of great importance to the study of climate change(Jiang et al., 2017). However, due to its harsh natural environment, conducting water resources surveys in the field is extremely labor-intensive and costly, and it is difficult to obtain stable long-term series data.

Satellite remote sensing technology provides a good solution to this problem. Landsat series images (Landsat 5, 7\&8) have high temporal resolution (16 days) and spatial resolution (30 m), also with high imaging quality, currently covering the widest range of time (1984-present). According to the spectral characteristics of water body, many water extraction indices have been developed through band operation, which provides convenience for water extraction from remote sensing images.

If we download huge amount of remote sensing images on TP covering long-time period first and then process them by software according to the traditional method, the work cycle will be greatly prolonged. What's more, for some large lakes, one image cannot completely cover them. At this time, two or more images are needed to extract the complete lakes, for which traditional methods are extremely cumbersome. As a cloud platform dedicated to data processing and analysis of geoscience, Google Earth Engine(GEE) supports online remote sensing image processing with many excellent remote sensing image processing algorithms. GEE not only saves the time of image downloading, but also shortens the work cycle greatly because of its powerful interaction ability and computational performance, so that remote sensing science has more time to analyze rather than waste in repeated processing. Through the friendly support of GEE for remote sensing image processing, we can easily and quickly obtain the vector information of water body, which provides a basis for further hydrological element extraction. In this paper, about 700 remote sensing images have been processed and analyzed.

\section{STUDY AREA, DATA SET AND PLATFORM}

\subsection{Study Area}

TP is the largest plateau in China and has the highest altitude in the world, which is known as "The Roof of the World" and "The Third Pole". It lies between $26^{\circ} 00^{\prime} \sim 39^{\circ} 47^{\prime}$ in the north latitude and $73^{\circ} 19^{\prime} \sim 104^{\circ} 47^{\prime}$ east longitude, as is shown in Figure 1.

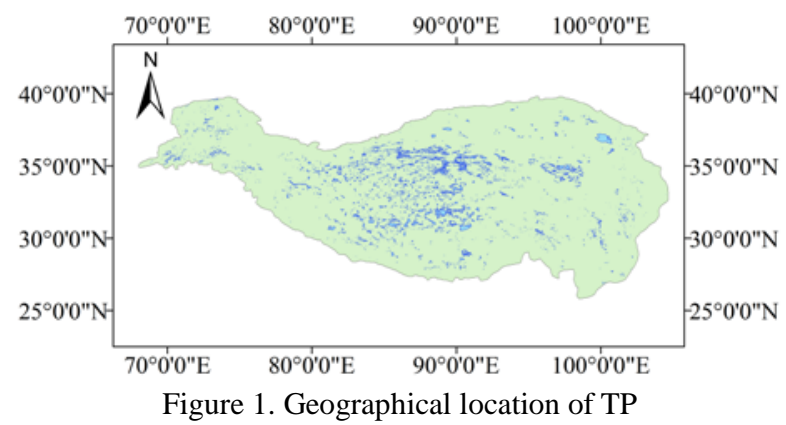




\subsection{Landsat Images}

Landsat5 TM, Landsat7 ETM+ and Landsat8 OLI are used herein, as listed in Table 1.

\begin{tabular}{|l|l|}
\hline Data & Year \\
\hline USGS Landsat 5 TM Collection 1 Tier 1 Raw & 1992-2011 \\
Scenes & \\
USGS Landsat 7 Collection 1 Tier 1 Raw & 2011-2013 \\
Scenes & \\
USGS Landsat 8 Collection 1 Tier 1 Raw & 2013-2016 \\
Scenes & \\
\hline
\end{tabular}

Table 1. Landsat dataset used in this study

Landsat Tier 1 includes Level-1 Precision Terrain (L1TP) processed data that have well-characterized radiometry and are inter-calibrated across the different Landsat sensors. The georegistration of Tier 1 scenes will be consistent and within prescribed tolerances [ $<=12 \mathrm{~m}$ root mean square error (RMSE)], that is, the registration accuracy reaches the sub-pixel level. Therefore, images of the Landsat can be used to extract water bodies and perform time series analysis.

\subsection{Processing Platform}

GEE is an open cloud platform developed by Google for geoscience data processing and analysis(Gorelick et al., 2017). It supports online programming and interactive display, and can process remote sensing data online without downloading images. Its powerful computing ability supports the processing of PB-level remote sensing data, making it suitable for large-scale and long-term series of research(Pekel et al., 2016). Moreover, the rich API documents of GEE absorb many superior remote sensing image processing algorithms, which are very friendly to people who focus on data processing rather than programming. The GEE interface is shown in Figure 2.

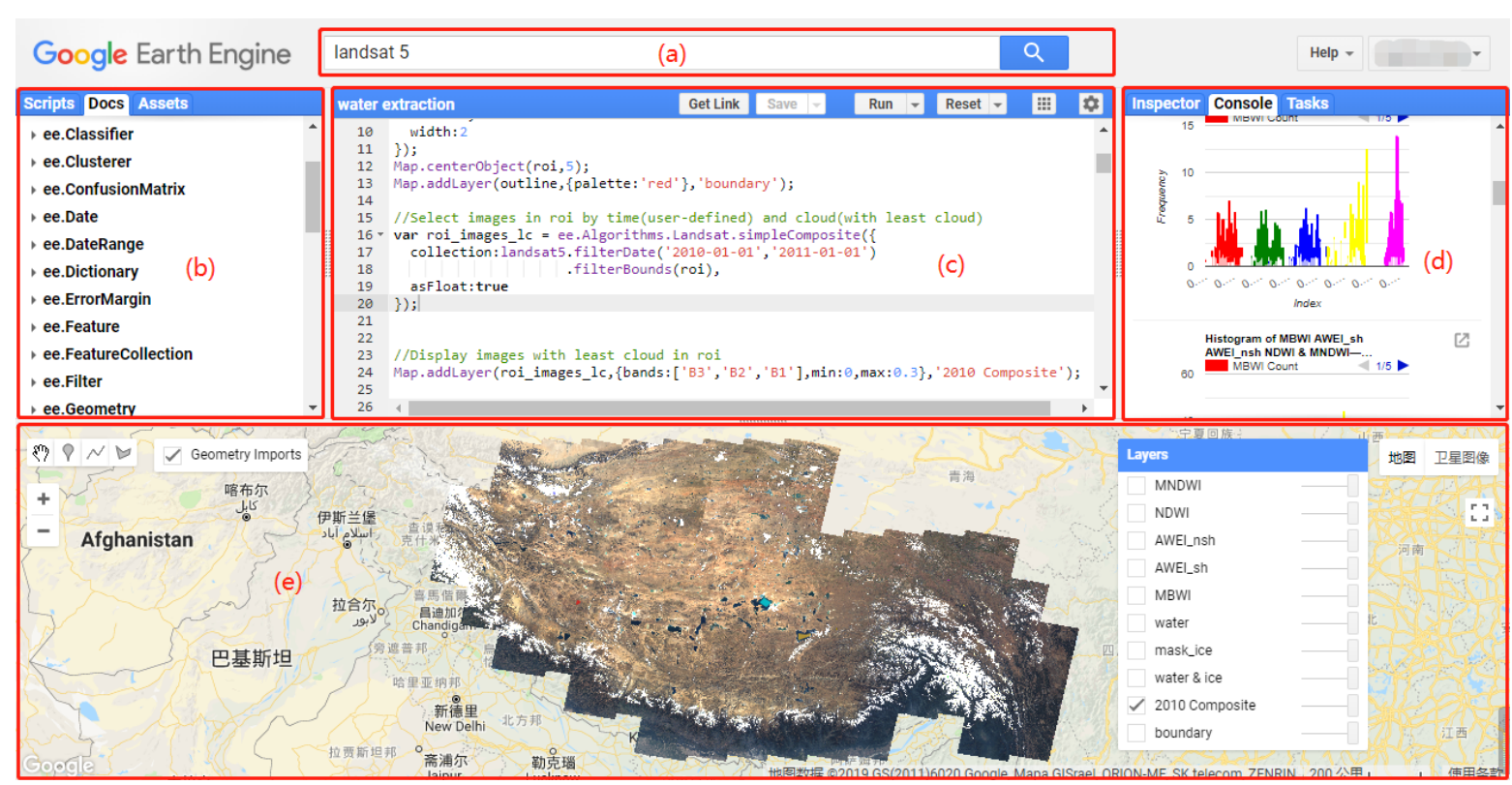

Figure 2. The interface of GEE: (a) is for data retrieval; (b) is for code preservation, document query and resource uploading; (c) is for code editing; (d) is for information query, result outputing, task downloading and running resource displaying; (e) is for layer displaying

\section{WATER EXTRACTION METHODS}

\subsection{Data Preprocessing}

Landsat5, 7 and 8 were de-clouded and radiometric calibrated. Cloud removal includes two methods: (1) Cloud cover sorting method based on image: sorting by cloud cover metadata and selecting images with cloud cover less than $20 \%$ to ensure image quality; (2) GEE algorithm based on pixels: it assigns a cloud score to each pixel and selects the lowest possible range of cloud scores at each point and then computes per-band percentile values from the accepted pixels. This algorithm also selects only the least-cloudy scenes in regions where more than maxdepth input scenes are available. The disadvantage of this method is that there will be obvious traces around the removed cloud patches, which will have a certain impact on the subsequent water extraction. Radiometric calibration converts the digital number(DN) value of the raw image into the top of atmosphere(TOA) value through the GEE algorithm.

However, Landsat5 images were only provided until 2011, and
Landsat8 images were provided from 2013, while there were wedge-shaped scan-to-scan gaps provided by Landsat 7 from 2011 to 2013. Therefore, gap-filling is necessary to use Landsat7 image. In this paper, the method of image fusion is used to fill the gaps, that is, two images with the closest time are fused to fill the missing bands.

\subsection{Water Extraction Index}

The construction of water index is based on the spectral characteristics of water, so that the calculated index can expand the difference between water and other objects, and then extract water according to the appropriate threshold. Normalized difference water index(NDWI), modified normalized difference water index(MNDWI), automated water extraction index(AWEI) (Feyisa et al., 2014) and multi-band water index(MBWI)(Wang et al., 2018), these water indices come from first to last, each with advantages and disadvantages, as listed from Eq1-Eq5. Variables in the Eqs are corresponding bands of Landsat images. 


$$
\begin{aligned}
\text { NDWI } & =\frac{\text { Green }- \text { NIR }}{\text { Green }+ \text { NIR }} \\
\text { MNDWI } & =\frac{\text { Green }- \text { SWIR1 }}{\text { Green }+ \text { SWIR1 }}
\end{aligned}
$$

AWEI_sh $=$ Blue $+2.5 \times$ Green $-1.5 \times($ NIR + SWIR1 $)-0.25 \times$ SWIR2

AWEI_nsh $=4 \times($ Green - SWIR1 $)-(0.25 \times$ NIR $+2.75 \times$ SWIR2 $)$

$$
\text { MBWI }=2 \times \text { Green }- \text { Red }- \text { NIR }- \text { SWIR1 - SWIR2 }
$$

\subsection{Selection of Water Index}

Sampling the rivers and lakes on TP, and the objects around the water that may be confused with, such as lands, shadows, ice and snow. The extraction effect of various water indices in complex environment is analyzed. Through trail and error, we found that AWEI and MBWI can achieve the best visual extraction effect for rivers and lakes, respectively.

The flow chart of this paper is as shown in Figure 3.

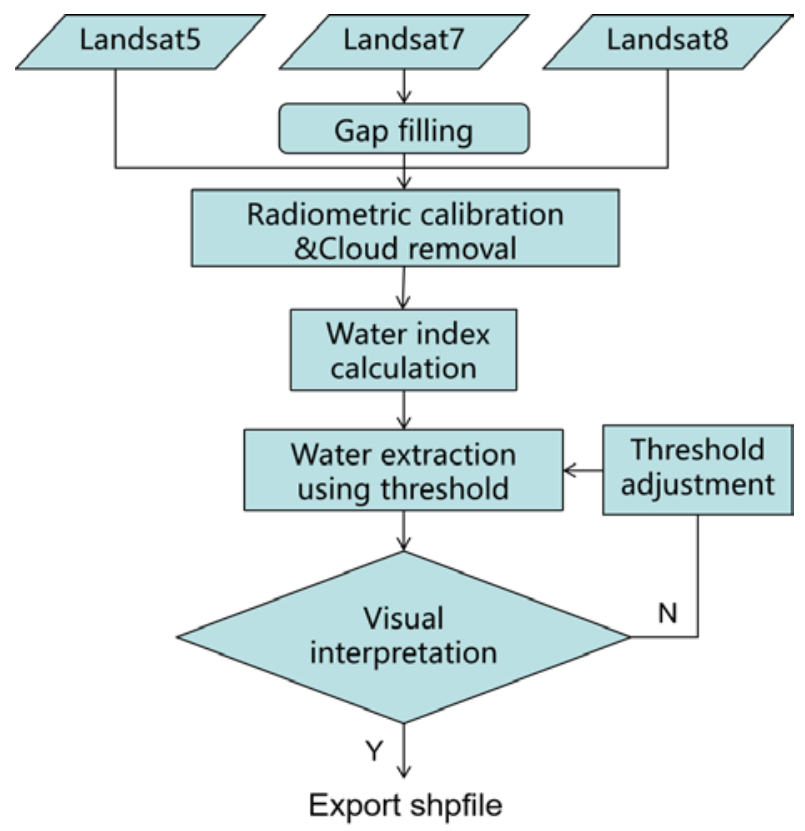

Figure 3. Flowchart for water extraction with Landsat images

\section{EXTRACTION RESULTS OF RIVERS AND LAKES}

When extracting rivers and lakes, buffer zones can be made

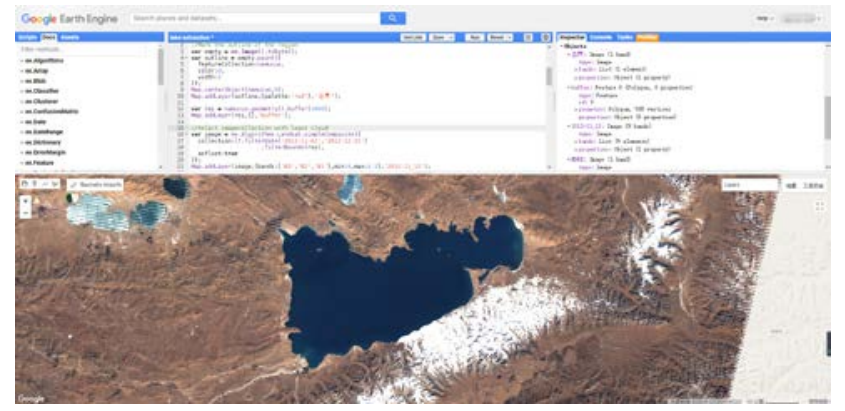

(a) Raw scene according to existing vectors or the range of water extraction can be artificially limited. On the one hand, disturbing objects can be eliminated, on the other hand, calculation efficiency can be improved. The extraction results of the Yarlung Zangbo River (taking the Nuxia Hydrological Station as an example) are as shown in Figure 4, and those of typical lakes (taking Nam Co as an example) are as shown in Figure 5. The Landsat7 image, which has been gap-filled, is used to extract Nam Co. It can be seen that it also has very good result.

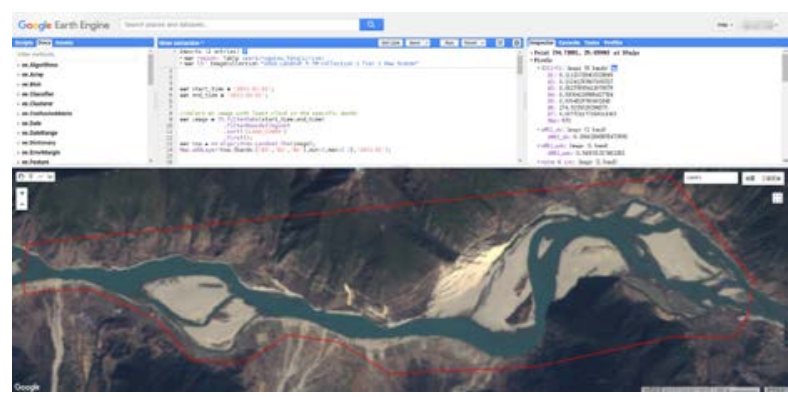

(a) Raw scene

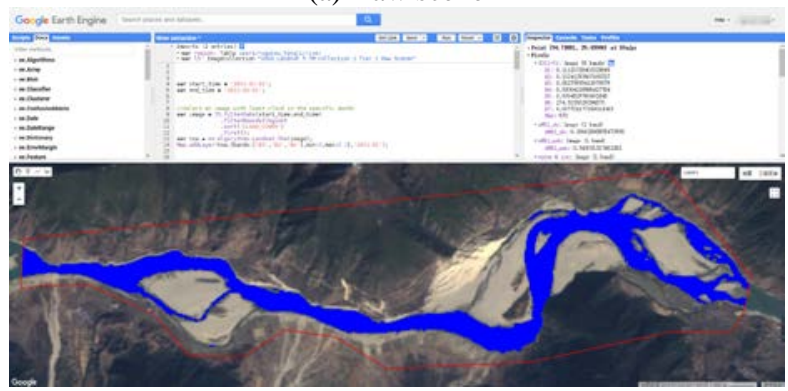

(b) Extraction result

Figure 4. Extraction of Yarlung Zangbo River reach

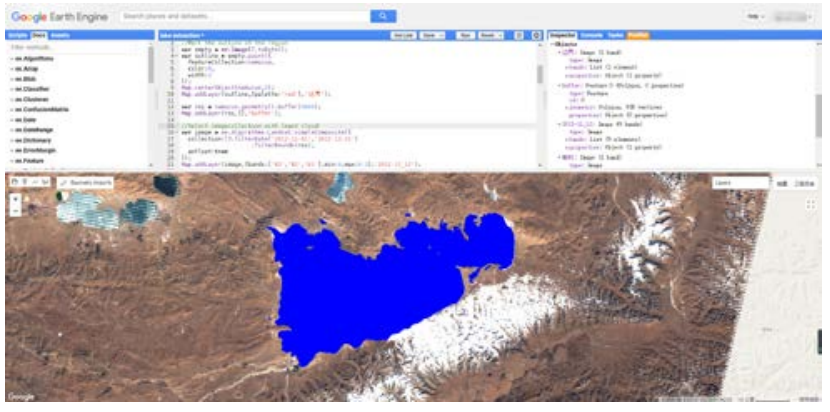

(b) Extraction result

Figure 5. Extraction of Nam Co with Landsat7 gap-filled images 
The area time series obtained from vector data are as shown in Figure 6 and Figure 7. Obviously, the monthly variation of river area in Figure 6 is periodic, as the annual variation trend is basically the same. As for the lake, shown in Figure 7, linear fitting shows that Ayukkumu Lake was in a rapid expansion state and it can be clearly seen in the vector map.Other typical lakes were also calculated but not shown.

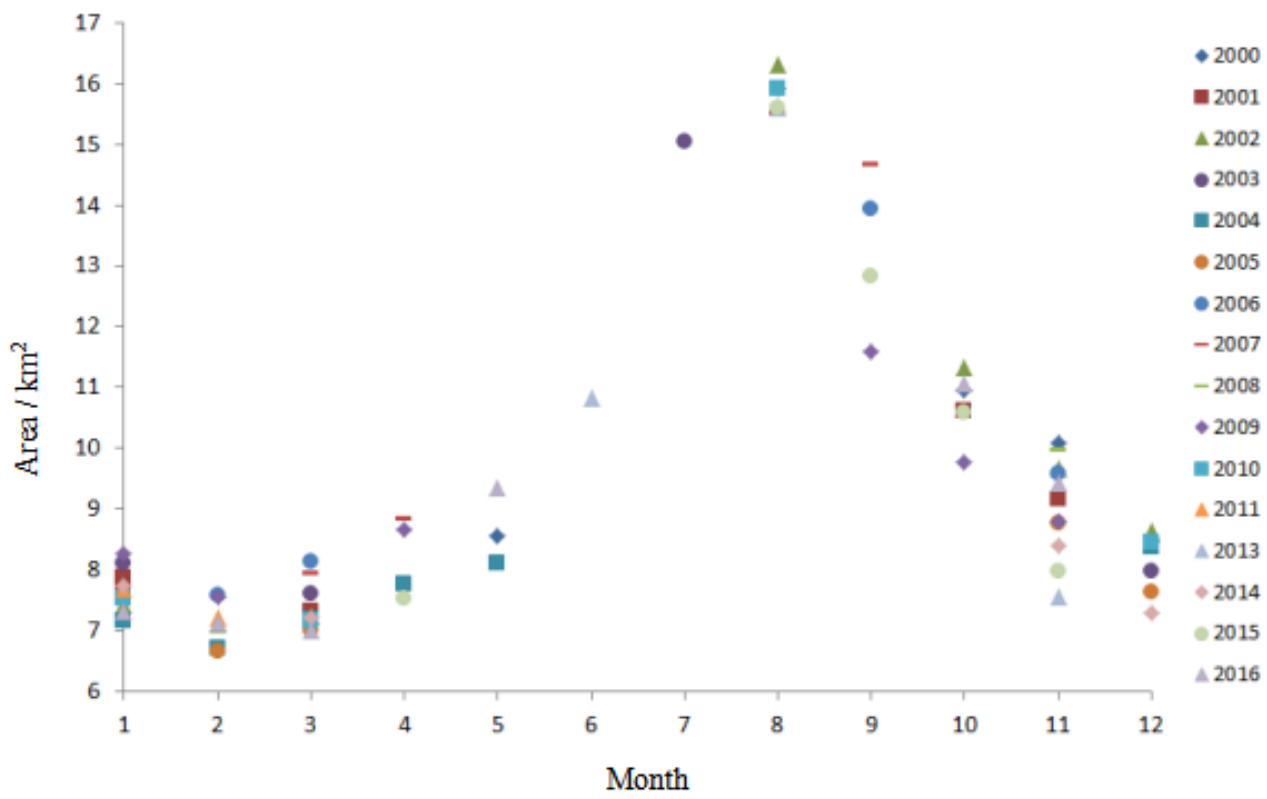

Figure 6. Monthly variation of river area at Nuxia Hydrological Station from 2000 to 2016
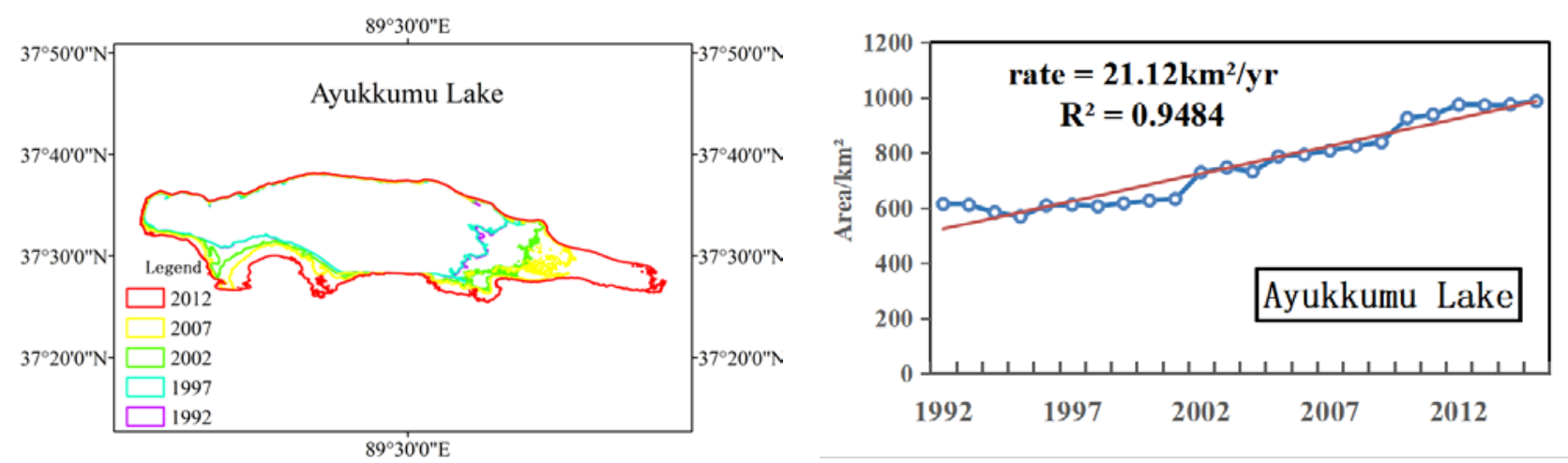

Figure 7. Annual area variation of several typical lakes (take the Ayukkumu Lake as an example) on TP from 1992 to 2015, left for the extraction results

\section{CONCLUSIONS}

This paper extracts rivers and lakes on TP based on GEE. The existing mainstream water index (NDWI, MNDWI) and the newly proposed water index (AWEI, MBWI) are sampled and validated. Different water indices are used to extract rivers and lakes according to local conditions, which can achieve good visual extraction results.

In view of the mountainous characteristics of the Yarlung Zangbo River, there are often a large number of mountain shadows. In addition to interference suppression by setting buffer zones, AWEI_sh and AWEI_nsh are used to effectively resist the impact of mountain shadows and cloud projection shadows, and achieve better extraction results. Typical lakes on TP are easier to extract than narrow rivers because of their large water area and mostly distributed in basin depressions. In this paper, MBWI is selected to extract lakes. On the one hand, MBWI has a stable threshold and little fluctuation; on the other hand, most lakes have freezing period in winter, and MBWI has a good effect on the extraction of ice lakes.
More than 150 Landsat images were used for the extraction of Yarlung Zangbo River monthly data from 2000 to 2016 and about 450 Landsat images were used to extract 20 typical lakes on TP for 23 years, from 1992 to 2015. The river area extracted by AWEI increases from February to August, decreases from August to next February, and keeps the same trend every year, which shows significant periodicity. However, the change in lake area varies, as Nam Co expand stably, the area of Qinghai Lake has a fluctuating growth, Ayukkumu Lake has a rapid expansion, while Peiku Co shrink at a very slow rate and so on.

With the friendly support of GEE for large data processing of remote sensing, the river and lake information of long time series on TP can be obtained efficiently, and the data set can be made to provide basic data for water resources change detection and various hydrological models on TP in futher study.

\section{ACKNOWLEDGEMENTS}

This research was supported by the National Science Foundation of China (91547210, 41771471, 41201425), the 
National Key Research and Development Program of China (2017YFA0603102), and the Fundamental Research Funds for the Central Universities.

\section{REFERENCES}

Feyisa, G.L., Meilby, H., Fensholt, R. and Proud, S.R., 2014. Automated Water Extraction Index: A new technique for surface water mapping using Landsat imagery. Remote Sensing of Environment, 140: 23-35.

Gorelick, N. et al., 2017. Google Earth Engine: Planetary-scale geospatial analysis for everyone. Remote Sensing of Environment, 202: 18-27.

Huang, Q. et al., 2018. An improved approach to monitoring Brahmaputra River water levels using retracked altimetry data. Remote Sensing of Environment, 211: 112-128.

Huang, Q. et al., 2018. Discharge estimation in high-mountain regions with improved methods using multisource remote sensing: A case study of the Upper Brahmaputra River. Remote Sensing of Environment, 219: 115-134.

Jiang, L., Nielsen, K., Andersen, O.B. and Bauer-Gottwein, P., 2017. Monitoring recent lake level variations on the Tibetan Plateau using CryoSat-2 SARIn mode data. Journal of Hydrology, 544: 109-124.

Pekel, J., Cottam, A., Gorelick, N. and Belward, A.S., 2016. High-resolution mapping of global surface water and its long-term changes. Nature, 540(7633): 418.

Wang, X. et al., 2018. A robust Multi-Band Water Index (MBWI) for automated extraction of surface water from Landsat 8 OLI imagery. International journal of applied earth observation and geoinformation, 68: 73-91. 